\title{
Coverage in sensor networks via persistent homology
}

\author{
VIN DE SILVA \\ ROBERT GHRIST
}

\begin{abstract}
We introduce a topological approach to a problem of covering a region in Euclidean space by balls of fixed radius at unknown locations (this problem being motivated by sensor networks with minimal sensing capabilities). In particular, we give a homological criterion to rigorously guarantee that a collection of balls covers a bounded domain based on the homology of a certain simplicial pair. This pair of (Vietoris-Rips) complexes is derived from graphs representing a coarse form of distance estimation between nodes and a proximity sensor for the boundary of the domain. The methods we introduce come from persistent homology theory and are applicable to nonlocalized sensor networks with ad hoc wireless communications.
\end{abstract}

$55 \mathrm{M} 25,93 \mathrm{~A} 15 ; 55 \mathrm{~N} 35$

\section{Introduction}

In topology, coverage problems associated to a collection of (say, convex) sets are classical and are classically handled by means of nerves and Čech theory; see Bott and $\mathrm{Tu}$ [3]. More recently, coverage problems have arisen in a variety of engineering contexts associated to networks, communication networks and sensor networks being prominent examples. Briefly, the sensor network version of the problem is as follows. Fix a domain $\mathcal{D}$ in Euclidean space. Given a collection of points in $\mathcal{D}$ (denoted "nodes"), assume each node's sensor "covers" a neighborhood of its location: for simplicity, a ball of fixed radius. One wants to know the extent of coverage by the nodes' sensor regions. In this paper we focus attention on the particular class of static, blanket coverage. By "static" is meant that the nodes are stationary; by "blanket" it is meant that one wants to determine if the entire domain $\mathcal{D}$ is contained in the union of sensor regions (closed balls) based at the nodes.

This problem is trivial if the location of the nodes is known (eg cell phone towers), and is both nontrivial and of critical importance if the locations of the nodes are not known (eg if the sensor nodes are small and/or "scattered" over the domain and acted on by environmental factors). 
We give an algebraic-topological criterion for certifying coverage based on a coarse notion of pairwise proximity between nodes, this being modeled on ad hoc wireless communication links between devices. The criterion is centralized (as opposed to distributed) and conservative (failure of the criterion does not imply failure of coverage). This coverage represents a novel application of classical (and fairly trivial) ideas in homology theory.

\subsection{Assumptions}

The methods we introduce are meant to work in settings where there are a large number of nodes with minimal and localized sensing capabilities. They have limited range and are devoid of localization and orientation capabilities, possessing merely a binary form of in-range distance measurement. More specifically, each node has a unique ID which it broadcasts. All other robots within range can "hear" their neighbor as either a strong or weak signal, depending on the distance to that node. We assume a small amount of information about the underlying domain $\mathcal{D} \subset \mathbb{R}^{d}$ : one knows only the dimension and connectivity and that the domain is not too "pinched" or "wrinkled". It is not necessary to assume knowledge of the topology of the domain, or of its large-scale geometry (eg volume).

The precise assumptions are as follows:

(A1) Nodes have radially symmetric coverage domains (a closed ball) of covering radius $r_{c}$.

(A2) Nodes broadcast their unique ID numbers. Each node can detect the identity of any node within radius $r_{s}$ via a strong signal, or via a weak signal within a larger radius $r_{w}$.

(A3) The radii of communication $r_{s}, r_{w}$ and the covering radius $r_{c}$ satisfy

$$
r_{c} \geq r_{s} / \sqrt{2} \quad \text { and } \quad r_{w} \geq r_{s} \sqrt{10}
$$

(A4) Nodes lie in a compact domain $\mathcal{D} \subset \mathbb{R}^{d}$. Nodes can detect the presence (but not the location or direction) of the boundary $\partial \mathcal{D}$ within a fence detection radius $r_{f}$.

(A5) The restricted domain $\mathcal{D}-N_{\widehat{r}}(\partial \mathcal{D})$ is connected, where

$$
N_{\widehat{r}}(\partial \mathcal{D})=\{x \in \mathcal{D}:\|x-\partial \mathcal{D}\| \leq \hat{r}\} \quad \text { and } \quad \hat{r}=r_{f}+r_{s} / \sqrt{2} .
$$

(Here $\|x-\partial \mathcal{D}\|$ denotes the distance from $x$ to the closest point of $\partial \mathcal{D}$.)

(A6) The fence detection hypersurface $\Sigma=\left\{x \in \mathcal{D}:\|x-\partial \mathcal{D}\|=r_{f}\right\}$ has internal injectivity radius at least $r_{s} / \sqrt{2}$ and external injectivity radius at least $r_{s}$. 
Assumptions (A1)-(A4) specify the communication and sensing capabilities of the nodes. Assumption (A5) is needed to prevent the domain from being too "pinched". This is clearly necessary since nodes with neither a map nor coordinates cannot distinguish between certain pinched domains and a disconnected domain. Assumption (A6) means that the outermost boundary cannot exhibit large-scale "wrinkling". This assumption is used in the details of the proof of Theorem 3.4 for eliminating pathological configurations. See Remark 4.5 for discussion on weakening this condition.

The last assumptions, (A5) and (A6), are the only restrictions on the geometry of the domain. We emphasize that the number of boundary components is not assumed to be known: nodes have no information about the boundary other than whether they are within range $r_{f}$. This $r_{f}$ is independent of the node-to-node communication radii $r_{s}$ and $r_{w}$ and the coverage radius $r_{c}$. The volume of the domain is not assumed to be known, and convexity is not at all required.

To summarize, the sensor data consists of three ingredients. Each node ascertains a primary list of node ID numbers associated to a "strong" detection signal, as well as a secondary list of node ID numbers flagged as coming from the "weak" signal. The third piece of data associated to each node is a fence-detection binary flag.

Remark 1.1 The numerical constants which appear in assumptions (A3), (A5) and (A6) are independent of the ambient dimension $d$. There are tighter constants which depend on $d$, and we summarize those results later on (Remark 4.3). Our proofs will be structured so that we can read off the improved constants without additional analysis. A resulting complication is that one special case (Remark 2.8) needs to be treated separately from the main argument.

\subsection{Results}

Topologists will not be surprised to learn that this coordinate-free data can be sufficient to rigorously verify coverage of the domain (ignoring regions too close to the boundary). Our strategy is as follows. The set of nodes $\mathcal{X}$ has a distinguished subset $\mathcal{X}_{f}$ of fence nodes, consisting of those nodes which lie within the fence detection radius $r_{f}$ of $\partial \mathcal{D}$. We build a nested collection of graphs:

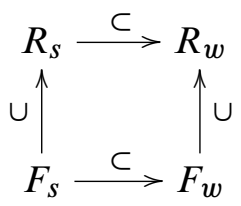

The graphs $R_{S}$ and $R_{w}$ are defined on $\mathcal{X}$ in the obvious manner via communication links: edges are present between nodes which are within distance $r_{s}$ and $r_{w}$ respectively. 
These are communication graphs for the strong and weak signals respectively. The graphs $F_{s}$ and $F_{w}$ are the strong and weak fence subgraphs - the maximal subgraphs of $R_{s}$ (respectively $R_{w}$ ) on the restricted set of vertices $\mathcal{X}_{f}$.

From these four graphs, we define a system of nested simplicial complexes

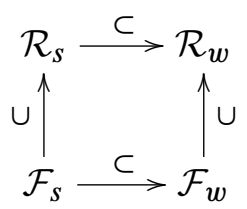

called Rips complexes, whose simplices are determined by "filling in" the corresponding graphs: each is the largest simplicial complex with the corresponding graph as its 1-d skeleton (ie the flag complex of the graph).

The sensor cover $\mathcal{U}$ is the union over $\mathcal{X}$ of discs of radius $r_{c}$. Our results link the topology of the cover $\mathcal{U}$ to the homology of the diagram of Rips complexes.

Main Theorem (Theorem 3.4) For a fixed set of nodes $\mathcal{X}$ in a domain $\mathcal{D} \subset \mathbb{R}^{d}$ satisfying assumptions (A1)-(A6), the sensor cover $\mathcal{U}$ contains $\mathcal{D}-N_{\widehat{r}}(\partial \mathcal{D})$ if the homomorphism

$$
\iota_{*}: H_{d}\left(\mathcal{R}_{s}, \mathcal{F}_{s}\right) \rightarrow H_{d}\left(\mathcal{R}_{w}, \mathcal{F}_{w}\right)
$$

induced by the inclusion $\iota$ : $\left(\mathcal{R}_{s}, \mathcal{F}_{s}\right) \hookrightarrow\left(\mathcal{R}_{w}, \mathcal{F}_{w}\right)$ is nonzero.

Increasingly, homology is a practical tools in applications which require computation of global structure: see the texts Hatcher [12] and Kaczynski, Mischaikow and Mrozek [14] for an introduction, the latter containing several current applications in science and engineering. Such applications include vision and recognition by Allili, Mischaikow and Tannenbaum [1], hybrid systems and control theory by Ames [2], rigorous verification of dynamics from experimental data by Mischaikow, Mrozek, Reiss and Szymczak [19], and global analysis of large data sets by the first author and Carlsson [5]. In the last example, there is a growing literature on the importance of persistent homology homology classes which persist as one changes a parameter in the system. It is this perspective that inspired the work in this paper.

\subsection{Related work}

The large literature on coverage problems for networks rests on two pillars of techniques. The first, the computational geometry approach, takes as its argument the geometric structure of the nodes - precise coordinates - and returns an auxiliary structure from which coverage or noncoverage can be deduced. Typical in this approach is the use 
of Delaunay triangulations (in 2-d); see eg Meguerdichian, Koushanfar, Potkonjak and Srivastava [18], Li, Wan and Frieder [16] and Zhang and Hou [23]. The precision with which coverage and lack of coverage can be determined is offset by the precision with which the coordinates of the nodes must be measured; such techniques are thus inapplicable in the context of coordinate-free sensors. The second approach uses probabilistic tools. Under assumptions of a uniform random distribution of points and a domain of known geometry, one can prove results about probability of coverage at a given density of nodes. Such methods are more appropriate in contexts for which the coordinates of nodes are unknown; see Koskinen [15], Xue and Kumar [22] and Liu and Towsley [17]. However, the assumptions on the uniformity of the distribution are crucial.

Recently, researchers have turned to deterministic approaches for coordinate-free sensor networks. The line of work begun by Rao et al [20] uses a heat flow to evolve node positions to virtual coordinates, using a set of fixed landmarks to frame the coordinates. These virtual coordinates appear useful in routing problems: they seem less useful in coverage problems, since there is no guarantee that the virtual coordinates are accurate. A recent use of computational geometry tools by Fekete et al [10] yields distributed algorithms for interrogating the topology of an unknown domain without information about node coordinates. These methods are capable of detecting which nodes are sufficiently within the "interior" of the coverage region.

In [6], the authors detail an approach to coverage in the case of coordinate-free sensors using homology of Rips complexes. The primary differences in [6] from the present work are as follows:

(1) Nodes communicate within a single fixed radius; the dual radii $r_{s}$ and $r_{w}$ are not used.

(2) There is a pre-established set of fence nodes which "know" that they lie exactly on the boundary of the domain $D$.

(3) The domain $\mathcal{D}$ is $2-\mathrm{d}$, simply connected, and polygonal; the boundary $\partial \mathcal{D}$ is canonically identified with the fence complex $\mathcal{F} \subset \mathcal{R}$ generated by the fence nodes.

The homology criterion in this setting of a $2-\mathrm{d}$ domain with controlled boundary is that the relative homology group $H_{2}(\mathcal{R}, \mathcal{F})$ has a generator $[\alpha]$ with $\partial \alpha \neq 0$. The proof is a simple application of exact sequences, owing to the fact that one has carefully constrained the nodes on $\partial \mathcal{D}$. The difficulty in the present paper is to manage arbitrary collections of nodes which can detect only proximity to the boundary $\partial \mathcal{D}$. The extension of the coverage criterion to domains of arbitrary dimensions is not inherently difficult, 
and results in a complication only when trying to compute optimal coefficients (see equation (4-1), for example).

\section{Factoring coverage through communication}

Given a collection of nodes $\mathcal{X}$ in a domain, we wish to determine the global properties of $\mathcal{U}$, the union of coverage domains centered at these nodes. However, we are constrained to use only communication connectivity data between nodes. Mathematically, this communication data takes the form of the 1-skeleta of the nerves of the covers of the node locations by balls of radius $r_{s}$ and $r_{w}$ respectively. In this section, we outline the basic constructions used to form a coverage criterion in the subsequent section.

\subsection{Simplicial complexes for covers}

The problem of computing the topological type of a union of sets is classical, and easily handled using the concept of a Cech complex (also called the nerve of the cover).

Definition 2.1 Given a collection of sets $\mathcal{U}=\left\{U_{\alpha}\right\}$, the $\check{C}$ ech complex of $\mathcal{U}, \mathcal{C}(\mathcal{U})$, is the abstract simplicial complex whose $k$-simplices correspond to nonempty intersections of $k+1$ distinct elements of $\mathcal{U}$.

If the cover is good - that is, if the cover sets and all nonempty finite intersections of cover sets are contractible - then the Čech complex $\mathcal{C}$ captures the topology of the cover: (see eg Hatcher [12])

Theorem 2.2 (The Čech Theorem) The Čech complex of a good cover has the homotopy type of the union of the cover sets.

Unfortunately, it is highly nontrivial to compute a Čech complex: one needs very precise data on pairwise distances between nodes. In the context of a sensor network with minimal range-sensing capabilities, the Čech complex is seemingly unattainable. Therefore, we consider the following related construction, which is more adapted to communication network constraints.

The Rips complex associated to a set of points is a notion originally developed by Vietoris in the earliest development of homology theory [21]. The concept was revived by Rips' work in geometric group theory and now generally goes by his name (see Gromov [11] and Hausmann [13]). 
Definition 2.3 Given a set of points $\mathcal{X}=\left\{x_{\alpha}\right\} \subset \mathbb{R}^{n}$ in Euclidean $n$-space and a fixed radius $\epsilon$, the Rips complex of $\mathcal{X}, \mathcal{R}_{\epsilon}(\mathcal{X})$, is the abstract simplicial complex whose $k$-simplices correspond to unordered $(k+1)$-tuples of points in $\mathcal{X}$ which are pairwise within Euclidean distance $\epsilon$ of each other.

Definition 2.4 In the setting of our main theorem, $\mathcal{R}_{s}, \mathcal{R}_{w}$ denote the Rips complexes on $\mathcal{X}$ with radii $r_{s}, r_{w}$ respectively.

The Rips complex is ideally suited to communication networks, since the entire complex is determined by pairwise communication data. Unfortunately, the Rips complex does not necessarily capture the topology of the union of cover discs: we have traded computability for accuracy. Figure 1 gives a fundamental class of examples for which the Rips complex fails to capture the Čech complex.
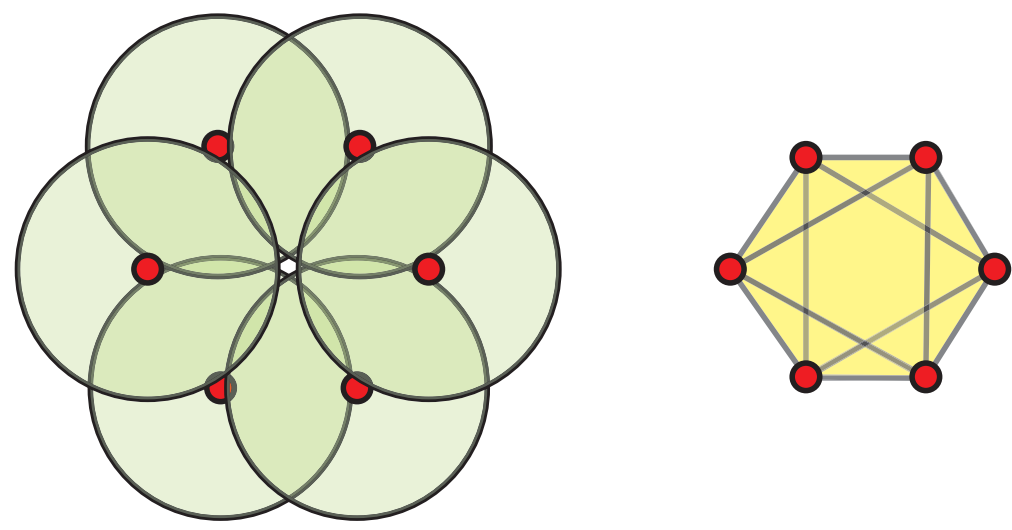

Figure 1: A class of examples for which the Rips complex $\mathcal{R}_{s}$ detects "phantom" topological features. Take $2 k+2$ points equidistributed on a circle of diameter $r_{s}+\lambda$ where $\lambda \ll 1$. The Čech complex (at the corresponding radius) is homotopy equivalent to a circle, as the Čech Theorem requires. The Rips complex however is isomorphic to the boundary of a cross-polytope in $k+1$ dimensions. This Rips complex is thus homeomorphic to the sphere $S^{k}$ and accordingly is very different from the Čech complex for $k>1$. Illustrated is the case $k=2$, with $\mathcal{R}_{s}$ an octahedron.

\subsection{Simplex geometry}

The constants relating the different radii in our theorems derive from the geometry of regular simplices. Let $\rho_{d}$ denote the circumradius of a regular $d$-simplex of unit edgelength. Then $\rho_{d}=\sqrt{d / 2(d+1)}$, which increases monotonically to a limit of $1 / \sqrt{2}$ 
as $d \rightarrow \infty$. This is the origin of the constant $1 / \sqrt{2}$ which appears in assumptions (A3), (A5) and (A6). The content of Theorem 2.5 is that regular simplices are worst-case for the covering problem, which is why the quantities $\rho_{d}$ are relevant.

\subsection{Optimal factorization of the Rips complex}

Since we assume that sensors can ascertain communication links, it follows that the 1-dimensional skeleton of the Čech complex can be determined directly from the sensor data. In the best of worlds, the Vietoris-Rips complex of the communication graph would suffice to capture the Čech complex: unfortunately, this is not true. However, one can squeeze the Čech complex (something not obtainable from the network graph alone) in between $\mathcal{R}_{s}$ and $\mathcal{R}_{w}$ (something determined by the communication graph and thus computable at the "hardware" level). In this subsection, we detail this nesting and prove optimality.

Theorem 2.5 Let $\mathcal{X}$ be a set of points in $\mathbb{R}^{d}$ and $\mathcal{C}_{\epsilon}(\mathcal{X})$ the Čech complex of the cover of $\mathcal{X}$ by balls of radius $\epsilon / 2$. Then there is chain of inclusions

$$
\mathcal{R}_{\epsilon}(\mathcal{X}) \subset \mathcal{C}_{\epsilon^{\prime}}(\mathcal{X}) \subset \mathcal{R}_{\epsilon^{\prime}}(\mathcal{X}) \quad \text { whenever } \quad \frac{\epsilon^{\prime}}{\epsilon} \geq \sqrt{\frac{2 d}{d+1}}=2 \rho_{d}
$$

Moreover, this ratio is the smallest for which the inclusions hold in general.

Proof The second inclusion is trivial because the criterion for inclusion of a simplex in $\mathcal{R}_{\epsilon^{\prime}}$ is weaker than the criterion for inclusion of a simplex in $\mathcal{C}_{\epsilon^{\prime}}$ (if the balls of radius $\epsilon^{\prime} / 2$ centered at the vertices have a common intersection then each pair of vertices is separated by distance at most $\epsilon^{\prime}$ ).

The first inclusion is equivalent to the following assertion: if a collection of points in $\mathbb{R}^{d}$ is such that every pair is separated by a distance at most $\epsilon$, then the balls of radius $\epsilon^{\prime} / 2$ centered on these points have a common intersection. Proving this for a set of $k+1$ points implies that every $k$-simplex of $\mathcal{R}_{\epsilon}$ belongs also to $\mathcal{C}_{\epsilon^{\prime}}$.

We will prove it first for a set of $d^{\prime}+1$ points $\left\{x_{0}, x_{1}, \ldots, x_{d^{\prime}}\right\}$, where $d^{\prime} \leq d$. Consider the function $f: \mathbb{R}^{d} \rightarrow \mathbb{R}$ defined as

$$
f(y)=\max _{0 \leq i \leq d^{\prime}}\left\|x_{i}-y\right\| .
$$

This is continuous and moreover $f(y) \rightarrow+\infty$ as $\|y\| \rightarrow \infty$, so it follows that $f$ has a global minimum $f\left(y_{0}\right)$, say. Define the critical vertices to be those points $x_{i}$ for which $\left\|x_{i}-y_{0}\right\|=f\left(y_{0}\right)$. 
Note that there is no vector $v$ which separates, in the sense that $v \cdot\left(x_{i}-y_{0}\right)>0$ for each critical vertex $x_{i}$. For such a vector we could calculate that

$$
\begin{aligned}
\left\|x_{i}-y_{0}\right\|^{2} & =\left\|x_{i}-\left(y_{0}+\lambda v\right)\right\|^{2}+2 \lambda v \cdot\left(x_{i}-y_{0}\right) \\
& >\left\|x_{i}-\left(y_{0}+\lambda v\right)\right\|^{2}
\end{aligned}
$$

for all $\lambda>0$ and therefore $f\left(y_{0}+\lambda v\right)<f\left(y_{0}\right)$ for $0<\lambda \ll 1$, contradicting minimality. Since no separating vector exists, $y_{0}$ must lie in the convex hull of the critical vertices.

It is convenient to make the translation $\hat{x}_{i}=x_{i}-y_{0}$. We can now find a convex combination $a_{0} \hat{x}_{0}+a_{1} \hat{x}_{1}+\cdots+a_{d^{\prime \prime}} \hat{x}_{d^{\prime \prime}}=0$ for some $d^{\prime \prime} \leq d^{\prime}$, after relabeling so that $x_{0}, x_{1}, \ldots, x_{d^{\prime \prime}}$ are critical vertices, the coefficients $a_{i}$ are strictly positive, and $a_{0}$ is the largest of the terms $a_{0}, a_{1}, \ldots, a_{d^{\prime \prime}}$. Then $-\hat{x}_{0}=\sum_{i=1}^{d^{\prime \prime}}\left(a_{i} / a_{0}\right) \hat{x}_{i}$ and so we have:

$$
-f\left(y_{0}\right)^{2}=-\left\|\hat{x}_{0}\right\|^{2}=\sum_{i=1}^{d^{\prime \prime}}\left(a_{i} / a_{0}\right) \hat{x}_{0} \cdot \hat{x}_{i}
$$

At least one of the $d^{\prime \prime}$ terms on the right-hand side must satisfy $\left(a_{i} / a_{0}\right) \hat{x}_{0} \cdot \hat{x}_{i} \leq$ $-f\left(y_{0}\right)^{2} / d^{\prime \prime}$, which can be weakened and rearranged to $f\left(y_{0}\right)^{2} / d \leq-\hat{x}_{0} \cdot \hat{x}_{i}$. We also know that $f\left(y_{0}\right)^{2}=\left\|\hat{x}_{0}\right\|^{2}=\left\|\hat{x}_{i}\right\|^{2}$. Putting this together,

and hence

$$
\begin{gathered}
f\left(y_{0}\right)^{2}(1+(2 / d)+1) \leq\left\|\hat{x}_{0}\right\|^{2}-2 \hat{x}_{0} \cdot \hat{x}_{i}+\left\|\hat{x}_{i}\right\|^{2} \\
=\left\|\hat{x}_{0}-\hat{x}_{i}\right\|^{2}=\left\|x_{0}-x_{i}\right\|^{2} \leq \epsilon^{2} \\
f\left(y_{0}\right) \leq \frac{\epsilon}{2} \sqrt{\frac{2 d}{d+1}} \leq \frac{\epsilon^{\prime}}{2} .
\end{gathered}
$$

It follows that the balls of radius $\epsilon^{\prime} / 2$ centered on the given $d+1$ points must meet at $y_{0}$.

For a set of greater than $d+1$ points, the result follows by applying Helly's theorem [8]. This asserts that a collection of $k \geq d+2$ convex sets in $\mathbb{R}^{d}$ has a nonempty common intersection provided only that the same is true for each subset of size $d+1$. If we have $k$ points spanning a simplex in $\mathcal{R}_{\epsilon}$, we have just established that each set of $d+1$ of the $\epsilon^{\prime} / 2$-balls at these vertices must have a nonempty intersection. By Helly's theorem, the same is true for the entire set of $k$ balls. Hence the vertices span a simplex in $\mathcal{C}_{\epsilon^{\prime}}$. The lower bound on $\epsilon^{\prime} / \epsilon$ is tight in the case of a regular $d$-simplex.

\subsection{Sensor complexes}

In order to determine coverage, it is necessary to know that there are no "holes"in the interior of the cover; as well, one must check that the cover extends sufficiently far out to the boundary of the domain. This latter condition prompts the following: 
Definition 2.6 Given a system with fence-detection as per assumption (A4), define the strong and weak fence subcomplexes, $\mathcal{F}_{s} \subset \mathcal{R}_{s}$ and $\mathcal{F}_{w} \subset \mathcal{R}_{w}$ respectively, to be the maximal subcomplexes of $\mathcal{R}_{s}$ and $\mathcal{R}_{w}$ whose vertices lie within distance $r_{f}$ of the $\partial \mathcal{D}$.

Lemma 2.7 Under the assumptions of our main theorem (in particular $r_{c} \geq r_{s} / \sqrt{2}$ ), any collection of nodes in $\mathcal{D}$ which form a simplex of $\mathcal{R}_{s}$ has its convex hull entirely contained within $\mathcal{U}$.

Remark 2.8 It follows from Lemma 2.7 that Theorem 3.4 is true in the trivial situation where $\mathcal{D}-N_{\widehat{r}}(\partial \mathcal{D})$ is entirely contained inside some $d$-simplex of $\mathcal{R}_{s}$.

The lemma can be read out of the following more precise result.

Lemma 2.9 Let $p$ belong to the convex hull of points $x_{0}, x_{1}, \ldots, x_{k} \in \mathbb{R}^{d}$ and suppose that $\left\|x_{i}-x_{j}\right\| \leq \epsilon$ for all $i, j$. Then $\left\|p-x_{i}\right\| \leq \epsilon \sqrt{2 d /(d+1)}=\epsilon \rho_{d}$, for some $i$.

Proof By Theorem 2.5, the balls of radius $\epsilon \rho_{d}$ centered at the points $x_{i}$ are guaranteed to meet at a common point $y$. Let $p=a_{0} x_{0}+a_{1} x_{1}+\cdots+a_{k} x_{k}$ be a convex combination of the nodes (so the $a_{i}$ are nonnegative and sum to unity). We rearrange this as $0=a_{0} \hat{x}_{0}+a_{1} \hat{x}_{1}+\cdots+a_{k} \hat{x}_{k}$ where $\hat{x}_{i}=x_{i}-p$. Taking the dot product with $\hat{y}=y-p$ we find that $0=a_{0} \hat{x}_{0} \cdot \hat{y}+a_{1} \hat{x}_{1} \cdot \hat{y}+\cdots+a_{k} \hat{x}_{k} \cdot \hat{y}$ so for some $i$ we must have $\hat{x}_{i} \cdot \hat{y} \leq 0$. In that case,

$$
\begin{aligned}
\left(\epsilon \rho_{d}\right)^{2} \geq\left\|x_{i}-y\right\|^{2}=\left\|\hat{x}_{i}-\hat{y}\right\|^{2} & =\left\|\hat{x}_{i}\right\|^{2}-2 \hat{x}_{i} \cdot \hat{y}+\|\hat{y}\|^{2} \\
& \geq\left\|\hat{x}_{i}\right\|^{2}=\left\|x_{i}-p\right\|^{2} .
\end{aligned}
$$

Remark 2.10 If $k<d$ then we can work in a $k$-dimensional affine subspace of $\mathbb{R}^{d}$ and conclude that $\left\|p-x_{i}\right\| \leq \epsilon \sqrt{2 k /(k+1)}=\epsilon \rho_{k}$ for some $i$.

\section{A homological criterion for coverage}

Our criterion for coverage is based on the homology of the inclusion map

$$
\iota:\left(\mathcal{R}_{s}, \mathcal{F}_{s}\right) \hookrightarrow\left(\mathcal{R}_{w}, \mathcal{F}_{w}\right) .
$$

We claim that coverage is implied by having a nonzero generator for the top-dimensional relative homology group $H_{d}\left(\mathcal{R}_{s}, \mathcal{F}_{s}\right)$ which persists (remains nonzero) under the induced homomorphism $\iota_{*}$ to $H_{d}\left(\mathcal{R}_{w}, \mathcal{F}_{w}\right)$.

Algebraic $8 \mathcal{G}$ Geometric Topology, Volume 7 (2007) 


\subsection{Intuition and persistence}

With the assumptions (A5) and (A6), the top dimensional relative homology group $H_{d}\left(\mathcal{D}, N_{\widehat{r}}(\partial \mathcal{D})\right)$ has rank one. Furthermore, $H_{d}\left(\mathcal{U} \cup N_{\widehat{r}}(\partial \mathcal{D}), N_{\widehat{r}}(\partial \mathcal{D})\right)$ is nonzero if and only if $\mathcal{U}$ contains $\mathcal{D}-N_{\widehat{r}}(\partial \mathcal{D})$. However, we cannot compute $\mathcal{U}$ directly. The simplicial complex which captures the topology of $\mathcal{U}$ - the Čech complex is impossible to compute without coordinates or at least accurate distances between sensors. Rips complexes are, in contrast, very manageable merely with communication data (and hence computable on the hardware level). Thus, it would make sense to hope that if $H_{d}\left(\mathcal{R}_{s}, \mathcal{F}_{s}\right)$ is nonzero, then $\mathcal{D}-N_{\widehat{r}}(\partial \mathcal{D}) \subset \mathcal{U}$.

But this is not always the case. Consider the 2-d setting of Figure 2, in which there is a cycle of points within $\mathcal{F}_{s}$ all of which are attached to a single vertex in $\mathcal{R}_{s}-\mathcal{F}_{s}$. This cycle is such that two of the edges are of length $r_{s}$, while the other two edges are of length $\epsilon \ll r_{s}$ As such, neither of the diagonals is of length $r_{s}$ and is therefore not present in $\mathcal{F}_{s}$. This system has $H_{2}\left(\mathcal{R}_{s}, \mathcal{F}_{s}\right) \neq 0$ : there exist "fake" relative 2-cycles which do not imply coverage of the entire domain. Other fake cycles can be generated from the examples of Figure 1 in any dimension.

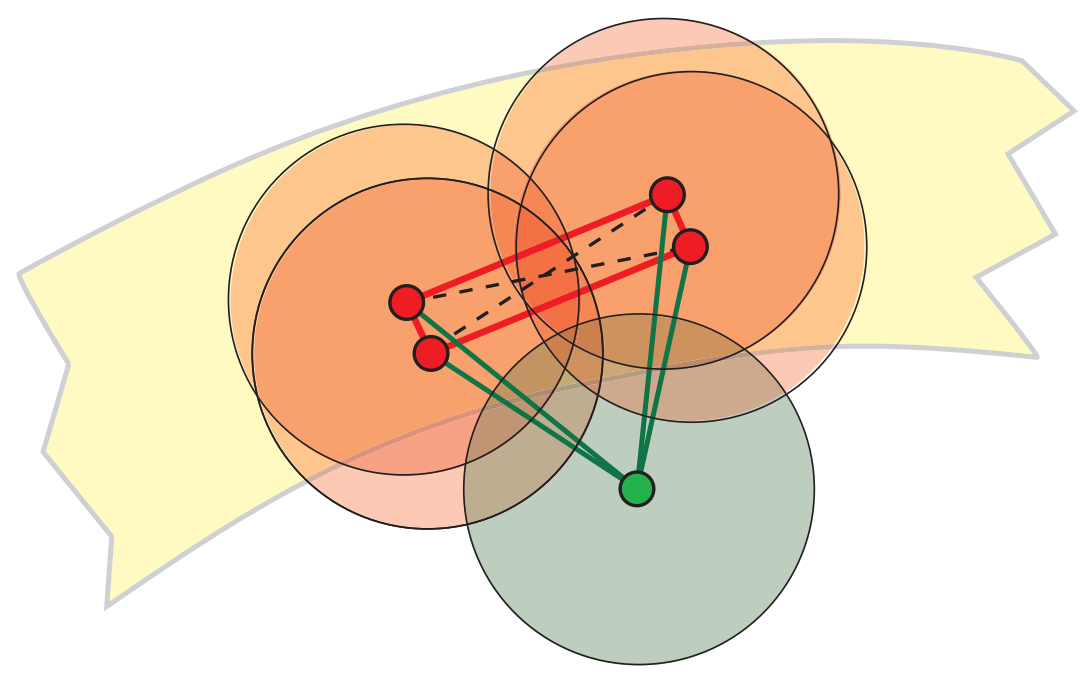

Figure 2: A fake generator for $H_{2}\left(\mathcal{R}_{s}, \mathcal{F}_{s}\right)$ which is annihilated by inclusion $\iota_{*}$ into $H_{2}\left(\mathcal{R}_{w}, \mathcal{F}_{w}\right)$. The strip illustrated is a collar of radius $r_{f}$.

Note, however, what happens to this relative 2-cycle under increasing the communication radius from $r_{s}$ to $r_{w}$, then the 1-cycle in $\mathcal{F}_{s}$ is "filled in" by diagonals, and the image of this fake class under $\iota_{*}$ is the zero element of $H_{2}\left(\mathcal{R}_{w}, \mathcal{F}_{w}\right)$. Assuming that 
these points are a portion of a larger subset of nodes, it is not necessarily the case that $H_{2}\left(\mathcal{R}_{w}, \mathcal{F}_{w}\right)=0$, since there may be a new fake 2-cycle which comes into existence at the longer communication lengths: but the original fake 2 -cycle is annihilated by $\iota_{*}$.

\subsection{Preliminary lemmas}

All of the difficulty in proving coverage comes from the analysis of the cover near the boundary $\partial \mathcal{D}$. For applications to sensor networks, we wish to minimize constraints on the number and types of boundary components. As a result, we can guarantee coverage only outside of a neighborhood of the boundary. We begin with some technical results concerning the geometry of how the fence nodes are situated.

Lemma 3.1 Let $\overline{N_{\widehat{r}}(\partial \mathcal{D})}=\mathbb{R}^{d}-\left(\mathcal{D}-N_{\widehat{r}}(\partial \mathcal{D})\right)$ denote the extended collar of $\mathcal{D}$. For any collection of nodes in $\mathcal{D}$ which form a simplex of $\mathcal{F}_{s}$, its convex hull lies within $\overline{N_{\widehat{r}}(\partial \mathcal{D})}$, or else we are in the trivial case described in Remark 2.8 .

The following proof is valid whenever $\hat{r} \geq r_{f}+r_{s} \sqrt{(d-1) / 2 d}=r_{f}+r_{s} \rho_{d-1}$.

Proof It suffices, by Carathéodory's Theorem [8], to show that the $d$-dimensional skeleton of $\mathcal{F}_{s}$ lies within $\overline{N_{\widehat{r}}(\partial \mathcal{D})}$. First consider the $(d-1)$-skeleton. For $k \leq d-1$ let $x_{0}, x_{1}, \ldots, x_{k} \in \mathcal{X}_{f}$ define a $k$-simplex $\sigma$ in $\mathcal{F}_{s}$. By Remark 2.10, for any $p \in \sigma$ there is an $x_{i}$ such that $\left\|x_{i}-p\right\| \leq r_{s} \rho_{d-1}$. Then

$$
\|p-\partial \mathcal{F}\| \leq\left\|x_{i}-\partial \mathcal{F}\right\|+\left\|p-x_{i}\right\| \leq r_{f}+r_{s} \rho_{d-1}
$$

so $p \in \overline{N_{\widehat{r}}(\partial \mathcal{D})}$.

Now suppose $p$ lies in some $d$-simplex $\sigma$ but is not in the $(d-1)$-skeleton. Then $\sigma$ is not degenerate, and $p$ must lie in its interior. Since $\mathcal{D}-\overline{N_{\widehat{r}}(\partial \mathcal{D})}=\mathcal{D}-N_{\widehat{r}}(\partial \mathcal{D})$ is connected and does not meet $\partial \sigma$, it is either entirely contained in the interior of $\sigma$ or it is disjoint from $\sigma$. Thus, either we are in the trivial situation of Remark 2.8, or $p \in \overline{N_{\widehat{r}}(\partial \mathcal{D})}$.

Remark 3.2 The proof is easier if we are not concerned with obtaining optimal dimension-dependent bounds. We can simply say that for any simplex $\sigma$ (regardless of its dimension) and $p \in \sigma$ there is a vertex $x_{i}$ such that $\left\|x_{i}-p\right\| \leq r_{s} / \sqrt{2}$. There is no need to discuss the $(d-1)$-skeleton or to mention Remark 2.8 .

The last and most technical lemma is a variant of Theorem 2.5 adapted to a $(d-1)-$ cycle in a thickened hypersurface in $\mathbb{R}^{d}$ of thickness $\Delta$. By this we mean a domain homeomorphic to a closed $(d-1)$-dimensional manifold cross an interval, which can be foliated by line segments of length no more than $\Delta$.

Algebraic 83 Geometric Topology, Volume 7 (2007) 
Lemma 3.3 Let $\mathcal{S} \subset \mathbb{R}^{d}$ be a thickened hypersurface of thickness $\Delta$ and let $\mathcal{X} \subset \mathcal{S}$ denote a collection of points which forms a $(d-1)-$ cycle $[\gamma] \in H_{d-1}\left(\mathcal{R}_{\epsilon}(\mathcal{X})\right)$, for some $\epsilon>0$ such that $\gamma$ is contained entirely within $\mathcal{S}$. If $[\gamma]=0$ in $H_{d-1}(\mathcal{S})$, then $[\gamma]=0$ in the $\epsilon^{\prime}$ Rips complex $\mathcal{R}_{\epsilon^{\prime}}(\mathcal{X})$, where

$$
\epsilon^{\prime}=\sqrt{\Delta^{2}+2 \epsilon^{2} \frac{d-1}{d}}=\sqrt{\Delta^{2}+\left(2 \rho_{d-1} \epsilon\right)^{2}} .
$$

Proof Denote by $\gamma$ the cycle as a geometric $(d-1)$-cycle in $\mathcal{S}$ and let $\mathcal{U}^{\prime}$ denote the union over $\mathcal{X}$ of balls of radius $\epsilon^{\prime} / 2$. For our choice of $\epsilon^{\prime}$ it follows from Remark 2.10 and Pythagoras' theorem that $\mathcal{U}^{\prime}$ contains the set $\mathcal{U}$ obtained by covering every point of $\gamma$ (simplices as well as vertices) with a ball of radius $\Delta / 2$.

Assume by way of contradiction that $[\gamma] \neq 0$ in $H_{d-1}\left(\mathcal{R}_{\epsilon^{\prime}}(\mathcal{X})\right)$ yet is trivial in $H_{d-1}(\mathcal{S})$. From Theorem 2.5, $\mathcal{R}_{\epsilon}(\mathcal{X}) \subseteq \mathcal{C}_{\epsilon^{\prime}}(\mathcal{X}) \subseteq \mathcal{R}_{\epsilon^{\prime}}(\mathcal{X})$. Thus, $\gamma$ is a nontrivial cycle in $\mathcal{C}_{\epsilon^{\prime}}(\mathcal{X})$. By the Čech Theorem and Alexander duality, there exists a point $p \in \mathcal{S}-\mathcal{U}^{\prime}$ enclosed by $\gamma$.

Since $\mathcal{S}$ has thickness $\Delta$, there is a line segment $\ell$ in $\mathcal{S}$ of length at most $\Delta$ passing through $p$ and connecting the two boundary components of $\mathcal{S}$. As $\gamma$ is trivial in $H_{d-1}(\mathcal{S})$, the two endpoints of $\ell$ are homologically not enclosed by $\gamma$, unlike $p$. For this reason $\ell$ must cross $\gamma$ at least once on each side of $p$. Thus $\ell$ intersects $\mathcal{U}$ in at least two disjoint segments. Each such segment has length at least $\Delta / 2$, which is a contradiction.

\subsection{The coverage criterion}

The following theorem is our principal coverage criterion:

Theorem 3.4 For a fixed set of nodes $\mathcal{X}$ in a domain $\mathcal{D} \subset \mathbb{R}^{d}$ satisfying assumptions (A1)-(A6), the sensor cover $\mathcal{U}$ contains $\mathcal{D}-N_{\widehat{r}}(\partial \mathcal{D})$ if the homomorphism

$$
\iota_{*}: H_{d}\left(\mathcal{R}_{s}, \mathcal{F}_{s}\right) \rightarrow H_{d}\left(\mathcal{R}_{w}, \mathcal{F}_{w}\right)
$$

induced by the inclusion $\iota: \mathcal{R}_{s} \hookrightarrow \mathcal{R}_{w}$ is nonzero.

Proof We may assume that we are not in the situation covered by Remark 2.8, where the theorem is trivially true.

Consider the simplicial realization map $\sigma: \mathcal{R}_{s} \rightarrow \mathcal{D}$ which sends vertices of $\mathcal{R}_{s}$ to the points $\mathcal{X} \subset \mathcal{D}$ and which sends a $k$-simplex of $\mathcal{R}_{s}$ to the (potentially degenerate) $k$-simplex given by the convex hull of the vertices implicated. Since the exceptional case is excluded, Lemma 3.1 implies that $\sigma$ takes the pair $\left(\mathcal{R}_{s}, \mathcal{F}_{s}\right)$ to $\left(\mathbb{R}^{d}, \overline{N_{\widehat{r}}(\partial \mathcal{D})}\right)$; 
we therefore construct the following diagram from the long exact sequences of the pairs:

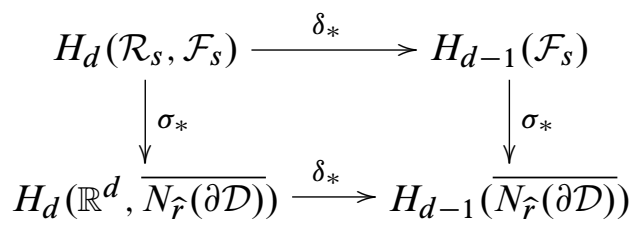

Here, $\delta_{*}$ acts on a class $[\alpha] \in H_{d}\left(\mathcal{R}_{s}, \mathcal{F}_{S}\right)$ by taking the boundary: $\delta_{*}[\alpha]=[\partial \alpha]$ in $H_{d-1}\left(\mathcal{F}_{s}\right)$. The diagram of (3-1) is commutative: $\delta_{*} \sigma_{*}=\sigma_{*} \delta_{*}$. The homology class $\sigma_{*} \delta_{*}[\alpha]$ measures the degree of $\partial \alpha$, or how many times the boundary of $\alpha$ "wraps around" the extended collar $\overline{N_{\widehat{r}}(\partial \mathcal{D})}$.

Now let $[\alpha] \in H_{d}\left(\mathcal{R}_{s}, \mathcal{F}_{s}\right)$ be a class for which $\iota_{*}[\alpha] \neq 0$.

Case $1 \sigma_{*} \delta_{*}[\alpha] \neq 0$.

By commutativity of $(3-1), \delta_{*} \sigma_{*}[\alpha]=\sigma_{*} \delta_{*}[\alpha] \neq 0$. Hence, $\sigma_{*}[\alpha] \neq 0$. Assume that $\mathcal{U}$ does not contain $\mathcal{D}-N_{\widehat{r}}(\partial \mathcal{D})$ and choose $p \in \mathcal{D}-\left(N_{\widehat{r}}(\partial \mathcal{D}) \cup \mathcal{U}\right)$. Since, by Lemma 2.7, every point in $\sigma\left(\mathcal{R}_{s}\right)$ lies within $\mathcal{U}$, this implies that $\sigma:\left(\mathcal{R}_{s}, \mathcal{F}_{s}\right) \rightarrow\left(\mathbb{R}^{d}, \overline{N_{\widehat{r}}(\partial \mathcal{D})}\right)$ factors through the pair $\left(\mathbb{R}^{d}-p, \overline{N_{\widehat{r}}(\partial \mathcal{D})}\right)$. However, $H_{d}\left(\mathbb{R}^{d}-p, \overline{N_{\widehat{r}}(\partial \mathcal{D})}\right)=0$ since, by Alexander duality, $H_{d}\left(\mathbb{R}^{d}-p, \overline{N_{\widehat{r}}(\partial \mathcal{D})}\right)=H^{0}\left(\mathbb{R}^{d}-\overline{N_{\widehat{r}}(\partial \mathcal{D})}, p\right)$, which vanishes since $\mathbb{R}^{d}-\overline{N_{\widehat{r}}(\partial \mathcal{D})}$ is connected. This gives the contradiction $\sigma_{*}[\alpha]=0$. Thus $\mathcal{U}$ contains $\mathcal{D}-N_{\widehat{r}}(\partial \mathcal{D})$ after all.

Case $2 \sigma_{*} \delta_{*}[\alpha]=0$.

We demonstrate that this case is impossible under the hypothesis $\iota_{*}[\alpha] \neq 0$. We construct the following commutative diagram with three rows, the top and bottom of which come from the long exact sequence of the pairs $\left(\mathcal{R}_{s}, \mathcal{F}_{s}\right)$ and $\left(\mathcal{R}_{w}, \mathcal{F}_{w}\right)$ respectively. The middle row comes from the pair $\left(\mathcal{R}_{m}, \mathcal{F}_{m}\right)$ - the Rips and Fence complexes computed at the "midrange" signal of radius

$$
r_{m}=r_{s} \sqrt{\frac{7 d-5+2 \sqrt{2 d(d-1)}}{2 d}}
$$

Algebraic 83 Geometric Topology, Volume 7 (2007) 
The inclusion map $\iota:\left(\mathcal{R}_{s}, \mathcal{F}_{s}\right) \hookrightarrow\left(\mathcal{R}_{w}, \mathcal{F}_{w}\right)$ factors through the pair $\left(\mathcal{R}_{m}, \mathcal{F}_{m}\right)$.

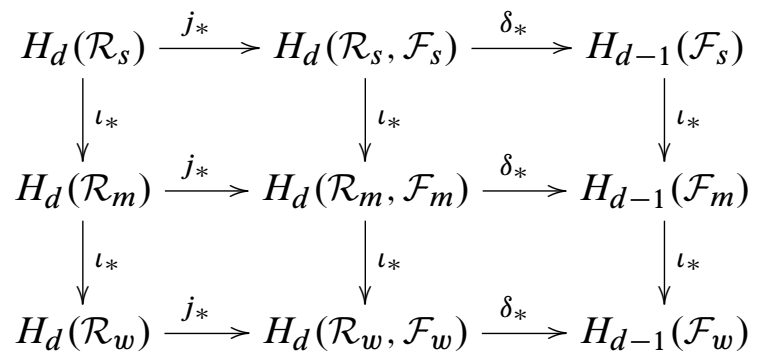

Represent the relative homology class $[\alpha]$ by an explicit cycle $\alpha$, comprised of simplices in $\mathcal{R}_{s}-\mathcal{F}_{s}$. We claim that the geometric $(d-1)$-cycle $\sigma(\partial \alpha)$ is contained in a particular shell $\mathcal{S}$, defined as follows. Let $\Sigma$ denote the hypersurface(s) of points at the precise fence detection radius:

$$
\Sigma=\left\{x \in \mathcal{D}:\|x-\partial \mathcal{D}\|=r_{f}\right\} .
$$

Let $\mathcal{S}$ denote the set of points in $\mathbb{R}^{d}$ within distance $r_{s} \sqrt{(d-1) / 2 d}$ of $\Sigma$ on the interior side (ie the side of $\Sigma$ corresponding to the interior of $\mathcal{D}$ ), and within distance $r_{s}$ of $\Sigma$ on the exterior side. It is helpful to define a signed distance function $|h(x)|=d(x, \Sigma)$ with $h(x)$ positive if and only if $x$ is on the exterior side; then $\mathcal{S}$ is defined by the inequalities $-r_{s} \sqrt{(d-1) / 2 d} \leq h(x) \leq r_{s}$.

Let $\sigma$ be a simplex in the geometric $(d-1)$-cycle $\partial \alpha$. Since $\sigma \in \mathcal{F}_{s}$, each vertex $x_{i}$ satisfies $h\left(x_{i}\right) \geq 0$. On the other hand, $\sigma$ is a face of some $d$-simplex $\tau \in \mathcal{R}_{s}-\mathcal{F}_{s}$ from the cycle $\alpha$. Since $\tau \notin \mathcal{F}_{S}$ the other vertex $y$ of $\tau$ must be on the interior side of $\Sigma$, so $h(y)<0$.

For all $p \in \sigma$ we have

$$
h(p) \leq h(y)+\|p-y\| \leq h(y)+\max _{i}\left(\left\|x_{i}-y\right\|\right)<0+r_{s}=r_{s} .
$$

Next, by Remark 2.10, we have $\left\|p-x_{i}\right\| \leq r_{s} \sqrt{(d-1) / 2 d}$ for some $i$. Therefore

$$
h(p) \geq h\left(x_{i}\right)-\left\|p-x_{i}\right\| \geq 0-r_{s} \sqrt{(d-1) / 2 d}=-r_{s} \sqrt{(d-1) / 2 d} .
$$

These inequalities prove that $\sigma \subset \mathcal{S}$; and in general this shows that the geometric realization of $\partial \alpha$ is entirely contained in $\mathcal{S}$.

From (A6), we know that $\mathcal{S}$ is a disjoint collection of thickened $(d-1)$-dimensional surfaces in $\mathbb{R}^{d}$ each of thickness at most

$$
\Delta=r_{s}\left(1+\sqrt{\frac{d-1}{2 d}}\right) .
$$

Algebraic ${ }^{3} \mathcal{G}$ Geometric Topology, Volume 7 (2007) 
Since $\sigma_{*} \delta_{*}[\alpha]=0$, we know that the cycle $\partial \alpha^{\prime}$ is nullhomologous within $\mathcal{S}$. Apply Lemma 3.3 with $\epsilon=r_{s}, \Delta$ as above, and

$$
\epsilon^{\prime}=\sqrt{\Delta^{2}+2 \epsilon^{2} \frac{d-1}{d}}=r_{s} \sqrt{\frac{7 d-5+2 \sqrt{2 d(d-1)}}{2 d}},
$$

to conclude that by increasing the radius from $r_{s}$ to $r_{m}$, the cycle $\partial \alpha^{\prime}$ becomes trivial. Hence, $\iota_{*} \delta_{*}[\alpha]=0 \in H_{d-1}\left(\mathcal{F}_{m}\right)$.

We may now rule out Case 2 as follows. By hypothesis, $[\alpha] \in H_{d}\left(\mathcal{R}_{s}, \mathcal{F}_{s}\right)$ is nonzero, as is $\iota_{*} \iota_{*}[\alpha] \in H_{d}\left(\mathcal{R}_{w}, \mathcal{F}_{w}\right)$. In the present case, $\iota_{*} \delta_{*}[\alpha]=0$ in $H_{d-1}\left(\mathcal{F}_{m}\right)$. Commutativity of (3-2) implies that $\delta_{*} \iota_{*}[\alpha]=0$. By exactness of this row, $\iota_{*}[\alpha]=j_{*}[\zeta]$ for some $[\zeta] \in H_{d}\left(\mathcal{R}_{m}\right)$. Theorem 2.5 implies that the map $\iota_{*}: H_{d}\left(\mathcal{R}_{m}\right) \rightarrow H_{d}\left(\mathcal{R}_{w}\right)$ factors through the homology of the Čech complex $\mathcal{C}_{w}=\mathcal{C}_{w}(\mathcal{X})$ of the cover of $\mathcal{X}$ with balls of radius $r_{w} / 2$ :

$$
\iota_{*}: H_{d}\left(\mathcal{R}_{m}\right) \rightarrow H_{d}\left(\mathcal{C}_{w}\right) \rightarrow H_{d}\left(\mathcal{R}_{w}\right) .
$$

From the Čech Theorem, $\mathcal{C}_{w}$ has the homotopy type of a subset of $\mathbb{R}^{d}$. Any such subset has vanishing homology in dimension $d$; hence $H_{d}\left(\mathcal{C}_{w}\right)=0$. We conclude that $\iota_{*}[\zeta]=0$. It follows from commutativity of (3-2) that

$$
0=j_{*}\left(\iota_{*}[\zeta]\right)=\iota_{*}\left(j_{*}[\zeta]\right)=\iota_{*}\left(\iota_{*}[\alpha]\right) \neq 0 .
$$

Contradiction. Case 2 is impossible under the assumption that

$$
r_{w} \geq r_{s}\left(\sqrt{\frac{2 d}{d+1}}\right)\left(\sqrt{\frac{7 d-5+2 \sqrt{2 d(d-1)}}{2 d}}\right)=r_{s} \sqrt{\frac{7 d-5+2 \sqrt{2 d(d-1)}}{d+1}}
$$

which is satisfied for any value of $d$ when, as in (A3), $r_{w} \geq r_{s} \sqrt{10}$.

\section{Remarks}

Remark 4.1 This is by no means a sharp criterion. It is first of all clearly possible to have the criterion always fail for injudicious choices of $r_{f}, r_{c}$, or $r_{w}$. For example, if $r_{w}$ is extremely large, then all nodes will be in (weak) communication and the complex $\mathcal{R}_{w}$ will be a single high-dimensional simplex with vanishing homology. Likewise, if $r_{c}$ is much larger than the bound in assumption (A3), then there will be many instances of coverage without a homological forcing.

This being said, we note that even if one chooses the minimal acceptable bounds from assumption (A3), it is still not hard to arrange the points to cover $\mathcal{D}-N_{\widehat{r}}(\partial \mathcal{D})$ without 
the homological criterion detecting this. The companion paper [6] gives a detailed examination in the single-radius case which is generally applicable in this setting.

Remark 4.2 The complexes $\left(\mathcal{R}_{m}, \mathcal{F}_{m}\right)$ used in case 2 of the proof of Theorem 3.4 are purely auxiliary: there is no need to ever compute these objects. They are required to determine the degree of the boundary of the relative cycle in the collar of the domain. As can be seen from the convolutions of the midrange signal construction, this is a delicate task.

Remark 4.3 One can improve the constants of assumptions (A3), (A5) and (A6) by using the expressions in the proof which depend on the dimension $d$. Specifically, we have:

(A3) The radii of communication $r_{s}, r_{w}$ and the covering radius $r_{c}$ satisfy

$$
r_{c} \geq r_{s} \sqrt{\frac{d}{2(d+1)}} \quad \text { and } \quad r_{w} \geq r_{s} \sqrt{\frac{7 d-5+2 \sqrt{2 d(d-1)}}{d+1}},
$$

where $d$ is the dimension of the domain.

(A5) The restricted domain $\mathcal{D}-N_{\widehat{r}}(\partial \mathcal{D})$ is connected, where

$$
N_{\widehat{r}}(\partial \mathcal{D})=\{x \in \mathcal{D}:\|x-\partial \mathcal{D}\| \leq \hat{r}\} \quad \text { and } \quad \hat{r}=r_{f}+r_{s} \sqrt{\frac{d-1}{2 d}} .
$$

(A6) The fence detection boundary $\left\{x \in \mathcal{D}:\|x-\partial \mathcal{D}\|=r_{f}\right\}$ has internal injectivity radius at least $r_{s} \sqrt{(d-1) /(2 d)}$ and external injectivity radius at least $r_{s}$.

For example, in the case $d=2$, the constants for (4-1) become:

$$
r_{c} \geq r_{s} \sqrt{\frac{1}{3}} \quad \text { and } \quad r_{w} \geq r_{s} \sqrt{\frac{13}{3}} .
$$

The latter inequality is a significant improvement over the $\sqrt{10}$ bound for arbitrary $d$.

Remark 4.4 We note that if the homological criterion is satisfied with a class $[\alpha]$ in $H_{d}\left(\mathcal{R}_{s}, \mathcal{F}_{s}\right)$, then the cover is generated only by the vertices implicated in the chain $\alpha$. Thus, by minimizing the choice of generator $\alpha$ within its persistent homology class, we can relax the redundancy of the cover. This has clear implications to issues of power conservation in sensor networks; see our paper [6] for details. 
Remark 4.5 The precise statement of (A6) in terms of injectivity radii requires the curve to be smooth. From the proof of Theorem 3.4, it is clear that the crucial condition is to have the shell $\mathcal{S}$ represent annular domains of thickness bounded by $\frac{3}{2} r_{s}$. In practice, having $\mathcal{D}$ piecewise-linear is admissible: even though the injectivity radii degenerate to zero, the set $\mathcal{S}$ is still an annular region(s) of width bounded by some larger length, depending on the sharpness of the curves. For a piecewise-linear $\partial \mathcal{D}$, an increase in $r_{w}$ based on the angle of the sharpest corner in the outermost boundary component makes the criterion rigorous.

Remark 4.6 The coverage criterion presented here is a very specific type of coverage: stationary blanket coverage. There are interesting questions involving, eg barrier coverage (in which one want the cover to separate a given domain) and sweeping coverage (in which the nodes move and "sweep" a cover over time). The paper [6] gives homological criteria for these settings and more in the simpler case of $d=2$ and controlled boundary nodes. We believe that the techniques of the current paper may be used to derive a persistent homology criterion applicable to these broader problems. The primary difficulty is in controlling what happens near the boundary of the domain.

Remark 4.7 We note that homology is (unlike homotopy groups!) computable, and that the coverage criterion of Theorem 3.4 can be checked in practice. We do not emphasize here the computational issues. Is suffices to note that we have used the computational homology software package Plex [4]. Simulations have been written using MATLAB as the frontend (primarily for generating the simplicial complexes from various point-data sets, and for data formatting and visualization.) The current implementation of Plex computes the dimensions of persistent homology groups (using algorithms as in Edelsbrunner, Letscher and Zomorodian [9] and Carlsson and Zomorodian [24]), which is enough to check whether the homomorphism $\iota_{*}$ in the criterion of Theorem 3.4 is nonvanishing.

\section{Acknowledgements}

The first and second authors are supported by DARPA; the second author is supported by the NSF. A preliminary version of these results appeared in the conference proceedings [7]. 


\section{References}

[1] M Allili, K Mischaikow, A Tannenbaum, Cubical homology and the topological classification of $2 D$ and $3 D$ imagery, from: "IEEE Intl. Conf. Image Proc." (2001) 173-176

[2] A Ames, A homology theory for hybrid systems: hybrid homology, Lect. Notes in Computer Science 3414 (2005) 86-102

[3] R Bott, L W Tu, Differential forms in algebraic topology, Graduate Texts in Mathematics 82, Springer, New York (1982) MR658304

[4] V de Silva, Plex Available at http://math.stanford.edu/comptop/programs/ plex/

[5] V de Silva, G Carlsson, Topological estimation using witness complexes, Symp. PointBased Graphics (2004)

[6] V de Silva, R Ghrist, Coordinate-free coverage in sensor networks with controlled boundaries, Int. J. Robotics Research 25 (2006) 1205-1222

[7] V de Silva, R Ghrist, A Muhammad, Blind swarms for coverage in 2-d, from: "Robotics: Systems and Science" (2005)

[8] J Eckhoff, Helly, Radon, and Carathéodory type theorems, from: "Handbook of convex geometry, Vol. A, B”, North-Holland, Amsterdam (1993) 389-448 MR1242986

[9] H Edelsbrunner, D Letscher, A Zomorodian, Topological persistence and simplification, from: "41st Annual Symposium on Foundations of Computer Science (Redondo Beach, CA, 2000)", IEEE Comput. Soc. Press, Los Alamitos, CA (2000) 454-463 MR1931842

[10] S Fekete, A Kröller, D Pfisterer, S Fischer, Deterministic boundary recognition and topology extraction for large sensor networks, from: "Algorithmic Aspects of Large and Complex Networks" (2006)

[11] M Gromov, Hyperbolic groups, from: "Essays in group theory", Math. Sci. Res. Inst. Publ. 8, Springer, New York (1987) 75-263 MR919829

[12] A Hatcher, Algebraic topology, Cambridge University Press, Cambridge (2002) MR1867354

[13] J-C Hausmann, On the Vietoris-Rips complexes and a cohomology theory for metric spaces, from: "Prospects in topology (Princeton, NJ, 1994)", Ann. of Math. Stud. 138, Princeton Univ. Press, Princeton, NJ (1995) 175-188 MR1368659

[14] T Kaczynski, K Mischaikow, M Mrozek, Computational homology, Applied Mathematical Sciences 157, Springer, New York (2004) MR2028588

[15] H Koskinen, On the coverage of a random sensor network in a bounded domain, from: "Proceedings of 16th ITC Specialist Seminar" (2004) 11-18 
[16] X-Y Li, P-J Wan, O Frieder, Coverage in wireless ad-hoc sensor networks, IEEE Transaction on Computers 52 (2003) 753-763

[17] B Liu, D Towsley, A study of the coverage of large-scale sensor networks, from: "IEEE International Conference on Mobile Ad-hoc and Sensor Systems" (2004)

[18] S Meguerdichian, F Koushanfar, M Potkonjak, M Srivastava, Coverage problems in wireless ad-hoc sensor network, from: "IEEE INFOCOM" (2001) 1380-1387

[19] K Mischaikow, M Mrozek, J Reiss, , A Szymczak, Construction of symbolic dynamics from experimental time series, Phys. Rev. Lett. 82 (1999) 1144

[20] A Rao, C Papadimitriou, S Shenker, I Stoica, Geographic Routing without Location Information, from: "Proceedings of 9th Annual International Conference on Mobile Computing and Networking (Mobicom'03)" (2003)

[21] L Vietoris, Über den höheren Zusammenhang kompakter Räume und eine Klasse von zusammenhangstreuen Abbildungen, Math. Ann. 97 (1927) 454-472 MR1512371

[22] F Xue, P R Kumar, The number of neighbors needed for connectivity of wireless networks, Wireless Networks 10 (2004) 169-181

[23] H Zhang, J Hou, Maintaining Coverage and Connectivity in Large Sensor Networks, from: "International Workshop on Theoretical and Algorithmic Aspects of Sensor, Ad hoc Wireless and Peer-to-Peer Networks", Florida (2004)

[24] A Zomorodian, G Carlsson, Computing persistent homology, Discrete Comput. Geom. 33 (2005) 249-274 MR2121296

Department of Mathematics and Computer Science, Pomona College

Claremont CA 91711, USA

Department of Mathematics and Coordinated Sciences Laboratory, University of Illinois

Urbana, IL 61801, USA

vin.desilva@pomona.edu, ghrist@math.uiuc.edu

Received: 25 November 2005 Revised: 29 January 2006 